\title{
ИМИТАЦИОННАЯ МОДЕЛЬ ОТОЖДЕСТВЛЕНИЯ ОБЪЕКТОВ ПРИ СТРУКТУРНО-СИСТЕМНОМ МОНИТОРИНГЕ ОБСТАНОВКИ
}

\author{
А. С. Литикова, С. Н. Разиньков \\ ВУНЦ ВВС «Военно-воздушная академия им. проф. Н. Е. Жуковского и Ю. А. Гагарина» (2. Воронеж)
}

Поступила в редакцию 26.02.2018 г.

\begin{abstract}
Аннотация. С использованием критерия минимума среднего риска в программной среде Qt Creator на языке C++ разработана имитационная модель отождествления объектов при структурно-системном мониторинге обстановки. На основе статистических экспериментов исследованы вероятности правильного и ложного отождествления объектов по оценкам угловых координат. Проведен анализ их зависимостей от среднеквадратических ошибок оценок параметров идентификации, размеров области размещения и числа объектов. Ключевые слова: критерий минимума среднего риска, апостериорная вероятность гипотезы об отождествлении, условная вероятность ситуации отождествления, вероятности правильного и ложного отождествления объектов.

Annotation. With use of criterion of a minimum of average risk in a program development environment Qt Creator in the C++ language the imitating model of identification of objects at structural and system monitoring of a situation is constructed. On the basis of statistical experiments probabilities of the correct and false identification of objects by estimates of angular coordinates are investigated. The analysis of their dependences on mean square errors of estimates of parameters of identification, number and the sizes of field of placement of objects is carried out. Keywords: criterion of minimum of average risk, posteriori probability of hypothesis of identification, conditional probability of situation of identification, probability of the correct and false identification of objects.
\end{abstract}

\section{ВВЕДЕНИЕ}

Для повышения достоверности оценки обстановки используются методы структурно-системного мониторинга, базирующиеся на совместной обработке данных (сведений) о контролируемых объектах, добываемых разнородными средствами. За счет комплексного анализа демаскирующих признаков целей парируются их пропуски в отдельных каналах поиска (наблюдения), в том числе обусловленные мерами маскировки и снижения заметности $[1,2]$.

При структурно-системном мониторинге обстановки требуется выполнять отождествление данных (сведений), поступающих от независимых источников [2]. Суть этой процедуры заключается в принятии решения

() Литикова А. С., Разиньков С. Н., 2018 о принадлежности признаков, выявленных различными средствами, одному и тому же объекту по результатам сопоставления параметров идентификации. Сопоставление параметров выполняется путем формирования списка соответствий между ними при минимизации потерь информации, соответствующих гипотезе об отождествлении [3].

В предлагаемой работе на основе критерия минимума среднего риска [3], обеспечивающего высокую надежность принимаемых решений при малом числе параметров идентификации [4], построена имитационная модель отождествления объектов с минимизацией вычислительных затрат на группирование однотипных признаков. По результатам ее статистических испытаний исследованы вероятности правильного и ложного отождествления объектов по результатам измерения угловых координат двумя пеленгаторными постами. 
Цель работы - анализ зависимостей показателей эффективности отождествления от среднеквадратических ошибок (СКО) определения однотипных параметров, размеров области размещения и числа объектов.

\section{ПОСТАНОВКА ЗАДАЧИ И РАЗРАБОТКА ИМИТАЦИОННОЙ МОДЕЛИ ОТОЖДЕСТВЛЕНИЯ ОБЪЕКТОВ}

Отождествление выполняется между $I$ и $J$ объектами, выявленными первым и вторым средствами мониторинга, путем нахождения наиболее близких по значениям параметров идентификации $\tilde{\theta}_{i}$ и $\hat{\theta}_{k}$, измеренных с СКО $\sigma_{1 \theta}$ и $\sigma_{2 \theta}$ соответственно. В общем случае, ввиду возможных пропусков объектов $I \neq J$.

В соответствии с критерием минимума среднего риска при отождествлении требуется установить принадлежность $I$ признаков, зафиксированных первым средством мониторинга, $\hat{E}=I+J$ объектам из числа $J$ вскрытых или $I$ пропущенных вторым средством мониторинга по правилу [3]

$$
\left(i^{\prime}, k^{\prime}\right)=\arg \min _{\substack{i=1 \ldots I, k=1 \ldots K}} \bar{R}_{p s},
$$

где

$$
\bar{R}_{p s}=\sum_{i=1}^{I} \sum_{k=1}^{K} R_{i k} P_{p s}^{i k},
$$

- средний риск принятия решения об отождествлении объектов, $R_{i k}$ и $P_{p s}^{i k}-$ риск и апостериорная вероятность гипотезы отождествления объектов с порядковыми номерами $i$ и $k, i=1, \ldots, I, k=1, \ldots, K$. В соответствии с байессовским подходом

$$
P_{p s}^{i k}=P_{a p}^{i} P_{k / i},
$$

где $P_{a p}^{i}$ - априорная вероятность условия отождествления $i$-го объекта, $i=1, \ldots, I, P_{k / i}$ условная вероятность ситуации отождествления $k$-го, $k=1, \ldots, K$, и $i$-го, $i=1, \ldots, I$, объектов $[3,4]$.

Значение $P_{k / i}, k=1, \ldots, K, i=1, \ldots, I$, находится как произведение вероятностей того, что при гипотезе отождествления пары объектов $(i, k)$ значение $i$-го, $i=1, \ldots, I$, признака принадлежит некоторой области значений, определяемой порогом принятия решения
[3], в окрестности значения $k$-го признака, $k=1, \ldots, K$, а значения признаков каждого $p$-го признака, $p \neq i, p=1, \ldots, I$, располагаются за ее пределами. Число возможных гипотез о принадлежности $i$-го признака, $i=1, \ldots, I$, одному из $K$ объектов определяется выражением [5]

$$
S=K^{I} \text {. }
$$

Полагая, что правильно принятые решения не приводят к информационным потерям, все ошибочные решения характеризуются одинаковыми рисками, а априорные вероятности условия отождествлении объектов равны, правило (1) с учетом (2), (3) представим в виде

$$
\left(i^{\prime}, k^{\prime}\right)=\arg \max _{\substack{i=1 . \ldots, k=1 \ldots K}} P_{k / i} .
$$

На основе (5) осуществляется оптимальный по критерию минимума среднего риска (1) выбор гипотезы о соответствии параметров идентификации с максимальной условной вероятностью ситуации отождествления. Однако при этом требуется провести сравнение значений $P_{k / i}, k=1, \ldots, K, i=1, \ldots, I$, для $\frac{K !}{I !(K-I) !}$ возможных сочетаний параметров. При большом числе контролируемых объектов указанная процедура затрудняет их отождествление в реальном масштабе времени.

Для повышения быстродействия алгоритма предлагается его квазиоптимальная модификация

$$
\left(i^{\prime}, k^{\prime}\right)=\left.\arg \max _{\substack{i=1 \ldots I, k=1 \ldots K}} P_{k / i}\right|_{P_{k / i}=0, P_{k^{\prime} i}=0} .
$$

Согласно (6), при отождествления объектов в матрице условных вероятностей $P_{k / i}$, $k=1, \ldots, K, i=1, \ldots, I$, находится наибольший элемент, соответствующий ситуации отождествления по $k^{\prime}$-му и $i^{\prime}$-му параметрам, $k^{\prime}=1, \ldots, K, i^{\prime}=1, \ldots, I$. Идентифицированную пару заносятся в список выполненных отождествлений, из матрицы условных вероятностей ситуаций отождествления исключаются элементы $k^{\prime}$-й строки и $i^{\prime}$-го столбца; в модифицированной матрице $P_{k / i}$ размером $(K-1) \times(I-1)$ снова осуществляется поиск наибольшего элемента; указанные процедуры 


\section{А. С. Литикова, С. Н. Разиньков}

повторяются I раз. Исключение соответствующих строк и столбцов из исходной матрицы на каждом этапе может выполняться путем обращения в нуль элементов $P_{k / i^{\prime}}$ и $P_{k^{\prime} / i ;}, k, k^{\prime}=1, \ldots, K, i, i^{\prime}=1, \ldots, I$.

При этом общее число операций по отождествлению объектов по сравнению с (5) сокращается в $L=\min (I, J)$ раз.

Правило (6) является квазиоптимальным ввиду возможных пропусков подлежащих идентификации объектов, поскольку в $k^{\prime}$-й строке и $i^{\prime}$-м столбце, исключающихся из матрицы условных вероятностей ситуаций отождествления, могут содержаться элементы, имеющие большие значения, чем оставшиеся в модифицированной матрице. В результате единичного ошибочного отождествления пары объектов $\left(k^{\prime \prime}, i^{\prime}\right)$ при их истинной идентификации $\left(k^{\prime}, i^{\prime}\right)$ и $\left(k^{\prime \prime}, i^{\prime \prime}\right)$ принятие правильного решения в отношении пары $\left(k^{\prime}, i^{\prime \prime}\right), k^{\prime}, k^{\prime \prime}=1, \ldots, K, i^{\prime}, i^{\prime \prime}=1, \ldots, I$, также становится невозможным.

С использованием взаимосвязей условных вероятностей ситуации отождествления объектов с СКО $\sigma_{1 \theta}$ и $\sigma_{2 \theta}$ для массивов значений $\tilde{\theta}_{i}$ и $\hat{\theta}_{k}, k=1, \ldots, K, i=1, \ldots, I$, в области $\Delta \theta$ [6] установлено, что при малом числе пропусков и высокой априорной точности оценки параметров идентификации [5] правило (6) может быть представлено в виде

$$
\begin{gathered}
\left(i^{\prime}, j^{\prime}\right)= \\
=\arg \min _{\substack{i=1 . \ldots I \\
j=1 . \ldots J}}\left[\frac{\left(\tilde{\theta}_{i}-\hat{\theta}_{k}\right)^{2}}{\sigma_{1 \sigma}^{2}+\sigma_{2 \sigma}^{2}}+\frac{1}{2} \ln \left(\sigma_{1 \sigma}^{2}+\sigma_{2 \sigma}^{2}\right)\right] .
\end{gathered}
$$

Второе слагаемое в квадратных скобках в правой части (7) ограничивает возможности группирования параметров идентификации, измеренных с низкой точностью.

На основе (7) в программной среде Qt Creator на языке C++ построена имитационная модель отождествления объектов по оценкам угловых координат $\tilde{\theta}_{i}$ и $\hat{\theta}_{j}, i=1, \ldots, I$, $j=1, \ldots, J$, в секторе углов $\Delta \theta$.

Генерация параметров идентификации выполнялась в приближении их распределения по нормальному закону с математическими ожиданиями, соответствующими истин- ным значениям угловых координат объектов, и дисперсиями $\sigma_{1 \sigma}^{2}$ и $\sigma_{2 \sigma}^{2}$ с применением датчика случайных чисел. Параметры идентификации и их оценки формировались независимо в каждой серии статистических испытаний модели.

Вероятность правильного отождествления объектов $P_{0}$ определялась как усредненное отношение числа правильно отождествленных параметров к общему количеству выполненных отождествлений, вероятность ложного отождествления $P_{1}$ - как отношение математического ожидания ложно выполненных к общему количеству возможных ошибочных отождествлений $[3,7]$.

\section{АНАЛИЗ ЭФФЕКТИВНОСТИ ОТОЖДЕСТВЛЕНИЯ ОБЪЕКТОВ ПРИ СТРУКТУРНО-СИСТЕМНОМ МОНИТОРИНГЕ ОБСТАНОВКИ}

На рис. 1 приведены зависимости вероятности правильного отождествления $I=20$ и $J=19$ объектов от СКО оценки их угловых координат одним из средств мониторинга $\sigma_{1 \theta}$ при фиксированной точности измерения данного параметра другим средством $\sigma_{2 \theta}=1^{\circ}$. Сплошной линией представлена закономерность изменения $P_{0}$, найденная при $\Delta \theta=180^{\circ}$, а штриховой линией - при $\Delta \theta=90^{\circ}$.

На рис. 2 приведены зависимости вероятности правильного отождествления объектов от СКО пеленгования $\sigma_{1 \theta}$ в секторе углов $\Delta \theta=90^{\circ}$ при $\sigma_{2 \theta}=1^{\circ}$ для вариантов: $I=15$ и $J=14$ (штриховая линия), $I=10$ и $J=9$ (сплошная линия).

Из представленных результатов следует, что при СКО $\sigma_{1 \theta}=0,5^{\circ}$ и $\sigma_{2 \theta}=1^{\circ}$ вероятность правильного отождествления 20 объектов в секторе углов $\Delta \theta=180^{\circ}$ составляет 0,85 , а вероятность ложного отождествления $P_{1}$ не превышает 0,01 . По мере увеличения $\sigma_{1 \theta}$ до $2^{\circ}$ величина $P_{0}$ снижается до 0,7 , а при $\sigma_{1 \theta}=5^{\circ}$ достигает 0,5 . С уменьшением сектора углов до $\Delta \theta=90^{\circ}$, приводящим к повышению плотности размещения объектов, вероятность их правильного отождествления при СКО $\sigma_{1 \theta}=0,5^{\circ}$ и $\sigma_{1 \theta}=5^{\circ}$ убывает до 0,7 и 0,3 соответственно. Данный эффект обусловлен пропусками под- 
Имитационная модель отождествления объектов при структурно-системном мониторинге ...

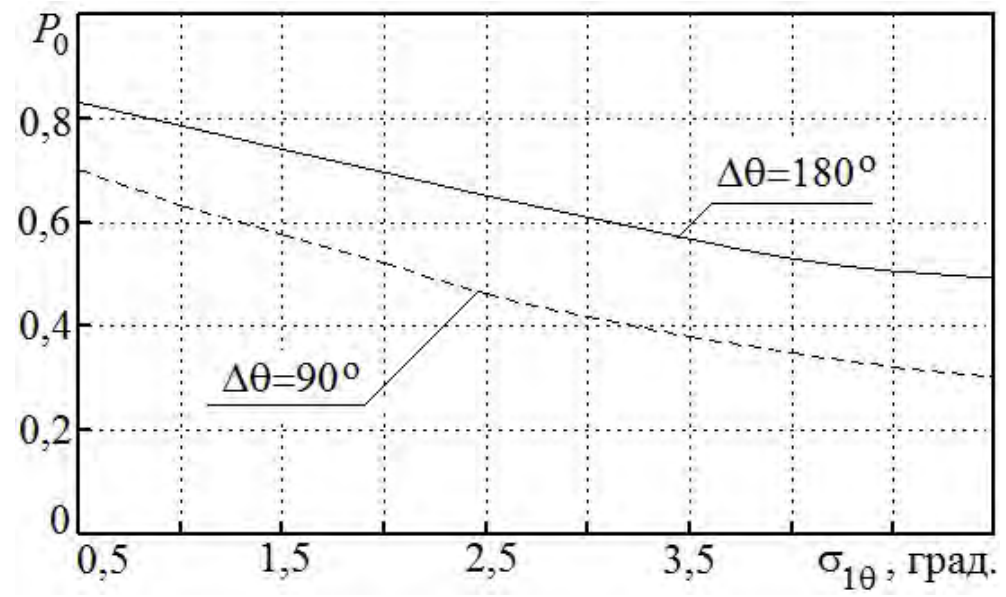

Рис. 1. Зависимости вероятности правильного отождествления $I=20$ и $\mathrm{J}=19$ объектов от СКО пеленгования $\sigma_{1 \theta}$

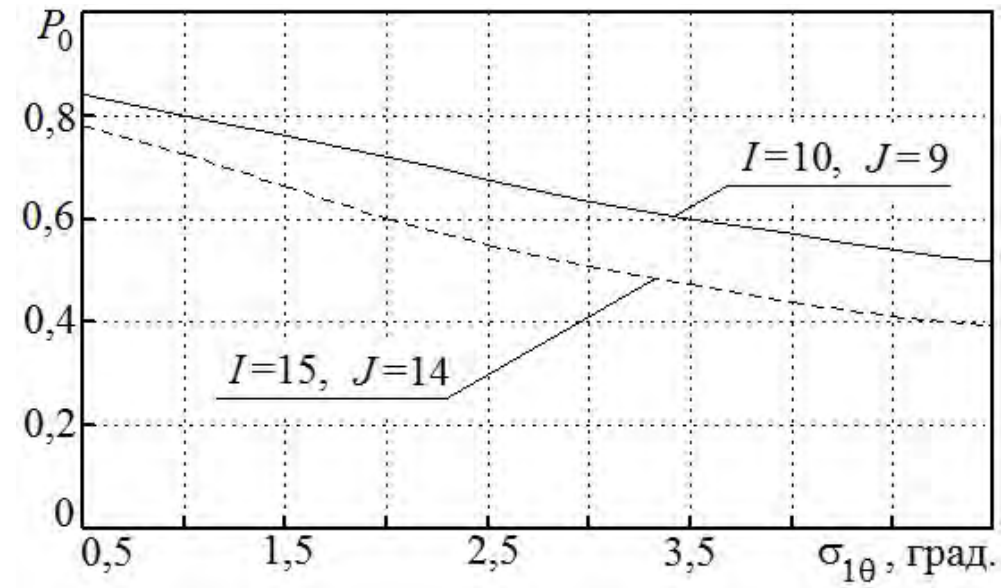

Рис. 2. Зависимости вероятности правильного отождествления объектов от СКО пеленгования $\sigma_{1 \theta}$ в секторе углов $\Delta \theta=90^{\circ}$

лежащих идентификации признаков в массивах значений $\tilde{\theta}_{i}$ и $\hat{\theta}_{j}, i=1, \ldots, I, j=1, \ldots, J$.

За счет сокращения числа объектов до 15 значение $P_{0}$ при $\sigma_{1 \theta}=5^{\circ}$ возрастает до 0,4 . Вероятность правильного отождествления $I=10$ объектов в секторе углов до $\Delta \theta=90^{\circ}$ при $\sigma_{1 \theta}=0,5^{\circ}$ составляет 0,85 , а при $\sigma_{1 \theta}=5^{\circ}$ снижается до 0,5 при $P_{1}=0,01$.

\section{ЗАКЛЮЧЕНИЕ}

На основе критерия минимума среднего риска в программной среде Qt Creator разработана имитационная модель отождествления объектов при структурно-системном мониторинге обстановки с минимизацией вариантов группирования параметров идентификации.
Показано, что при равенстве нулю рисков принятия правильных решений и одинаковых информационных потерях для всех неправильных решений, а также равных априорных вероятностях условий отождествления идентификации подлежат объекты с максимальной условной вероятностью ситуации отождествления. При малом числе пропусков и высокой априорной точности оценки параметров идентификации отождествление может быть выполнено путем группирования объектов с минимальными значениями квадратов разностей однотипных признаков, нормированных на суммарную дисперсию их измерений.

На основе статистических испытаний модели исследованы вероятности правильного и ложного отождествления объектов по оценкам угловых координат. Выявлены зако- 


\section{А. С. Литикова, С. Н. Разиньков}

номерности изменения показателей эффективности отождествления объектов по мере увеличения их числа, размеров области размещения и СКО оценок параметров идентификации.

\section{СПИСОК ЛИТЕРАТУРЫ}

1. Меньшаков, Ю. К. Теоретические основы технических разведок / Ю. К. Меньшаков. М. : МГТУ имени Н.Э. Баумана, 2008. - 536 с.

2. Радиоэлектронные системы: основы построения и теория. Справочник / Под ред. Я. Д. Ширмана. - М. : Радиотехника, 2007. $512 \mathrm{c}$.

3. Машков, Г. М. Статистические критерии и показатели качества отождествления локационных объектов / Г. М. Машков // Известия вузов. Радиоэлектроника. - 2001. - Т. 44, № 9. - С. 40-48.

Литикова Александра Сергеевна - младший научный сотрудник Научно-исследовательского испытательного института (радиоэлектронной борьбы), Военный учебно-научный центр Военно-воздушных сил «Военно-воздушная академия имени профессора Н. Е. Жуковского и Ю. А. Гагарина» (г. Воронеж).

Тел.: 8-919-180-52-29

E-mail: litikova-as@mail.ru

Разиньков Сергей Николаевич - ведущий научный сотрудник Научно-исследовательского испытательного института (радиоэлектронной борьбы), Военный учебно-научный центр Военно-воздушных сил «Военно-воздушная академия имени профессора Н. Е. Жуковского и Ю. А. Гагарина» (г. Воронеж).

Тел.: 8-904-212-71-79

E-mail: razinkovsergey@rambler.ru
4. Барабаш, Ю. Л. Вопросы статистической теории распознавания / Ю. Л. Барабаш, Б. В. Варский, В. Т. Зиновьев // Под ред. Б. В. Варского. - М. : Советское радио, 1967. $400 \mathrm{c}$.

5. Школьныци, Л. А. Отождествление РЭС на основе данных от разнородных датчиков информации / Л. А. Школьный, С. И. Ясько // Радиотехника. - 1999. - № 9. - С. 3-7.

6. Попова, О. Э. Отождествление объектов в системах активно-пассивной радиолокации / О. Э. Попова, С. Н. Разиньков // Измерительная техника, 2008. - № 6. - С. 43-48.

7. Разиньков, С. Н. Оптимальное и квазиоптимальное отождествление объектов при структурно-системном мониторинге обстановки / С. Н. Разиньков, Е. А. Решетняк // Физика волновых процессов и радиотехнические системы. - 2015. - Т. 18, № 3. - С. 42-47.

Litikova Alexandra S. - junior research assistant of the Research test center of radio-electronic struggle, Air Force Academy named after Professor N. E. Zhukovsky and Yu. A. Gagarin (Voronezh).

Tel.: 8-919-180-52-29

E-mail: litikova-as@mail.ru

Razinkov Sergey N. - lead research assistant of the Research test center of radio-electronic struggle, Air Force Academy named after Professor N. E. Zhukovsky and Yu. A. Gagarin (Voronezh). Tel.: 8-904-212-71-79

E-mail: razinkovsergey@rambler.ru 